\title{
Erodibilidade de alguns solos do Estado do Amazonas
}

Guido Ranzani ( ${ }^{*}$ )

\section{Resumo}

A presença de dados morfológicos e analíticos de grandes grupos de solos do Estado do Amazonas permitiu a avaliação de sua erodibilidade, empregando um método nomográfico. Os resultados são sugestivos de índices de erodibilidade maiores nos solos Podzolizados e menores nos solos Latossólicos. O Latossolo Vermelho Amarelo apresentou índices de erodibilidade muito baixos em toda a extensão do perfil. O Latossolo Amarelo apresentou menores índices apenas nos horizontes superficiais.

\section{INTRODUÇÃO}

A resistência que um solo oferece à erosão representa um atributo de grande importância e de cujo conhecimento depende a possibilidade de escolha das alternativas de uso e práticas conservacionistas mais recomendáveis às terras cultivadas .

Dados referentes à erodibilidade dos solos podem ser obtidos com um conhecimento seguro da natureza das chuvas, condições de relevo e da superfície do solo, através do emprego de coletores e canteiros com diferentes declividades e submetidos a diferentes conjuntos de práticas agrícolas.

A erodibilidade do solo pode ainda ser avaliada com auxílio dos dados morfológicos e analíticos de perfís de solo e o objetivo deste trabalho é aplicar este método em alguns solos do Estado do Amazonas.

Wischmeier et al. (1971) idealizaram um sistema nomográfico para avaliação da erodibilidade dos solos. Os parâmetros por eles utilizados foram: 1) porcentagem de limo mais porcentagem de areia muito fina, 2) porcentagem de areia com diâmetro maior do que $0,1 \mathrm{~mm}, 3$ ) porcentagem de matéria orgânica, 4) estrutura e 5) permeabilidade do solo.
Valores do índice de erodibilidade já foram obtidos para solos dos Estados Unidos (Wischmeier \& Smith, 1965), de Porto Rico (Smith \& Abruna, 1955) e do Estado de São Paulo (Freire \& Pessotti, 1974).

\section{MATERIAL E MÉTODO}

O método utilizado para obtenção do índice de erodibilidade dos solos do Estado do Amazonas é o nomográfico de Wischmeier et al. (1971).

Os solos cujos resultado's morfológicos e analíticos se prestaram para a avaliação do índice $(K)$ de erodibilidade, foram selecionados dos trabalhos indicados no Quadro 1.

Os intervalos de valores do índice de erodibilidade podem definir cinco classes de erodibilidade, a saber :

\begin{tabular}{|c|c|}
\hline Muito alta & $\mathrm{K}$ superior a 0,50 \\
\hline Alta & $\mathrm{K}$ entre 0,35 e 0,50 \\
\hline Média & $\mathrm{K}$ entre 0,25 e 0,35 \\
\hline Baixa & $\mathrm{K}$ entre 0,10 e 0,25 \\
\hline Muito baixa & $K$ inferior a 0,10 \\
\hline
\end{tabular}

Para melhor visualizar as tendências de cada solo, foi estimada a freqüência das classes através do porcentual de ocorrência dos valores K (Quadro 4).

O teor de matéria orgânica foi avaliado para os primeiros $20 \mathrm{~cm}$ de profundidade do solo.

\section{RESUltados E DISCUSSÃo}

No Quadro 2 são apresentados os resultados obtidos sobre as amostras superficiais de solos do Estado do Amazonas.

A freqüência das classes de erodibilidade em termos do porcentual de ocorrência é indicada no Quadro 3.

(") - Instituto Nacional de Pesquisas da Amazônia, Manaus. 
QUADRO 1 - Solos utilizados e referências bibliográficas.

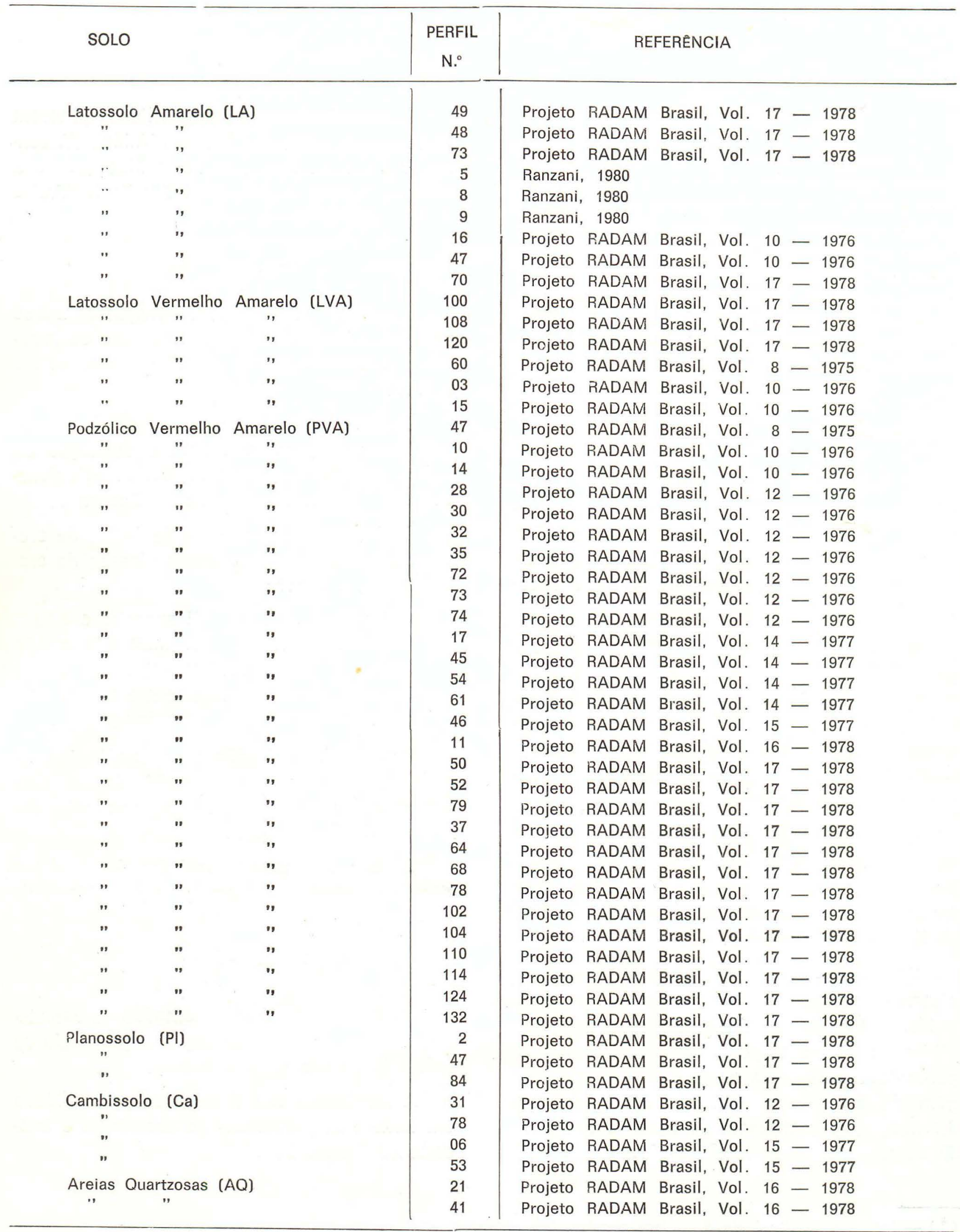




\begin{tabular}{|c|c|c|c|c|c|c|c|}
\hline \multicolumn{3}{|c|}{ SOLO } & PERFIL & \multicolumn{4}{|c|}{ REFERENCIA } \\
\hline$"$ & $"$ & " & 69 & Projeto & RADAM & Brasil, Vol. 17 & -1978 \\
\hline$"$ & $"$ & , & 16 & Projeto & RADAM & Brasil, Vol. 14 & -1977 \\
\hline \multicolumn{3}{|c|}{ Alúvios (Al) } & 14 & Projeto & RADAM & Brasil, Vol. 15 & -1977 \\
\hline \multicolumn{3}{|c|}{ 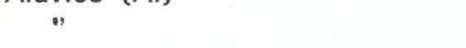 } & 15 & Projeto & RADAM & Brasil, Vol. 15 & -1977 \\
\hline \multicolumn{3}{|l|}{$r$} & 22 & Projeto & RADAM & Brasil, Vol. 15 & -1977 \\
\hline Glei & Pouco $\mathrm{H}$ & Húmico (GPH) & 48 & Projeto & RADAM & Brasil, Vol. 10 & -1976 \\
\hline & & " & 71 & Projeto & RADAM & Brasil, Vol. 12 & -1976 \\
\hline$"$ & & $"$ & 05 & Projeto & RADAM & Brasil, Vol. 15 & -1977 \\
\hline$"$ & " & $"$ & 20 & Projeto & RADAM & Brasil, Vol. 15 & -1977 \\
\hline$"$ & $"$ & $"$ & 6 & Projeto & RADAM & Brasil, Vol. 17 & -1978 \\
\hline$"$ & $"$ & $"$ & 86 & Projeto & RADAM & Brasil, Vol. 17 & -1978 \\
\hline$"$ & $"$ & $"$ & 11 & Projeto & RADAM & Brasil, Vol. 15 & -1977 \\
\hline$"$ & $"$ & $"$ & 13 & Projeto & RADAM & Brasil, Vol. 15 & -1977 \\
\hline$"$ & " & $"$ & 45 & Projeto & RADAM & Brasil, Vol. 15 & -1977 \\
\hline$"$ & $"$ & $"$ & 04 & Projeto & RADAM & Brasil, Vol. 15 & -1977 \\
\hline \multirow{2}{*}{\multicolumn{3}{|c|}{ Concrecionário Laterítico (CL) }} & 82 & Projeto & RADAM & Brasil, Vol. 12 & -1976 \\
\hline & & & 66 & Projeto & RADAM & Brasil, Vol. 17 & -1978 \\
\hline \multicolumn{3}{|c|}{ Laterita Hidromórfica (LH) } & 45 & Projeto & RADAM & Brasil, Vol. 10 & -1976 \\
\hline \multicolumn{3}{|c|}{ " " } & 03 & Projeto & RADAM & Brasil, Vol. 16 & -1978 \\
\hline \multicolumn{3}{|c|}{$"$} & 12 & Projeto & RADAM & Brasil, Vol. 16 & -1978 \\
\hline \multicolumn{3}{|c|}{$"$} & 19 & Projeto & RADAM & Brasil, Vol. 15 & -1977 \\
\hline \multicolumn{3}{|c|}{$"$} & 25 & Projeto & RADAM & Brasil, Vol. 15 & -1977 \\
\hline " & & $"$ & 58 & Projeto & RADAM & Brasil, Vol. 17 & -1978 \\
\hline
\end{tabular}

QUADRO 2 - Dados e resultados dos solos do Estado do Amazonas.

\begin{tabular}{|c|c|c|c|c|c|c|}
\hline Solo & Município & $\begin{array}{c}\text { Silte } \\
+ \\
\text { a.m.f. }\end{array}$ & $\begin{array}{c}\text { Areia } \\
> \\
0,10 \mathrm{~mm}\end{array}$ & $\begin{array}{c}\text { Matéria } \\
\text { Orgânica } \\
\%\end{array}$ & $\begin{array}{c}\text { Estrutura } \\
\left.\qquad{ }^{*}\right]\end{array}$ & $\begin{array}{c}\text { Indice } \\
\text { de } \\
\text { Erodib. }\end{array}$ \\
\hline LA & Novo Aripuanã & 31,6 & 31,4 & 4,6 & Gr. peq. & 0,09 \\
\hline$"$ & Manicoré & 18,1 & 62,2 & 5,5 & $"$ & 0,08 \\
\hline$"$ & Novo Aripuanã & 33,7 & 39,8 & 4,6 & $"$ & 0,16 \\
\hline$"$ & Manaus & 9,5 & 64,2 & 3,8 & $"$ & 0,08 \\
\hline$"$ & Manaus & 18,0 & 19,7 & 7,0 & $"$ & 0,07 \\
\hline$"$ & Manaus & 20,0 & 10,0 & 5,7 & $"$ & 0,07 \\
\hline$"$ & Nhamundá & 14,8 & 9,4 & 4,8 & $"$ & 0,08 \\
\hline$"$ & Itacoatiara & 3,4 & 70,5 & 2,2 & $"$ & 0,08 \\
\hline$"$ & Novo Aripuanã & 34,7 & 6,8 & 5,7 & $"$ & 0,08 \\
\hline LVA & Manicoré & 16,1 & 44,0 & 2,7 & $"$ & 0,14 \\
\hline$"$ & Novo Aripuanã & 12,2 & 78,8 & 2,8 & $"$ & 0,10 \\
\hline$"$ & Novo Aripuanã & 22,3 & 14,8 & 4,6 & $"$ & 0,07 \\
\hline$"$ & Rio Marauiá & 32,5 & 42,5 & 2,2 & $"$ & 0,15 \\
\hline$"$ & Urucará & $17,(3$ & 30,8 & 3,4 & $"$ & 0,09 \\
\hline$"$ & Macauari & 3,9 & 60,7 & 2,7 & $"$ & 0,08 \\
\hline PVA & Missão Toototobi & 34,5 & 48,5 & 2,1 & $"$ & 0,17 \\
\hline$"$ & Airão & 49,0 & 32,0 & 2,5 & $"$ & 0,20 \\
\hline$"$ & Urucará & 22,0 & 53,0 & 3,2 & $"$ & 0,09 \\
\hline$"$ & Pauini & 43,0 & 37,0 & 1,8 & $"$ & 0,25 \\
\hline$"$ & Pauini & 59,0 & 28,0 & 1,7 & $"$ & 0,42 \\
\hline$"$ & Boca do Acre & 36,5 & 41,5 & 0,9 & $"$ & 0,21 \\
\hline$"$ & Boca do Acre & 55,0 & 33,0 & 2,5 & $"$ & 0,33 \\
\hline$"$ & Boca do Acre & 72,0 & 20,0 & 3,1 & $"$ & 0,39 \\
\hline$"$ & Lábrea & 31,0 & 58,0 & 3,0 & Gr. sples & 0,14 \\
\hline$"$ & Lábrea & 61,5 & 12,5 & 1,7 & Gr. peq. & 0,33 \\
\hline
\end{tabular}


QUADRO 2 - (Contínuação).

\begin{tabular}{|c|c|c|c|c|c|c|}
\hline Solo & Município & $\begin{array}{c}\text { Silte } \\
+ \\
\text { a.m.f. }\end{array}$ & $\begin{array}{c}\text { Areia } \\
> \\
0,10 \mathrm{~mm}\end{array}$ & $\begin{array}{c}\text { Matéria } \\
\text { Orgânica } \\
\%\end{array}$ & $\begin{array}{c}\text { Estrutura } \\
*\end{array}$ & $\begin{array}{c}\text { Indice } \\
\text { de } \\
\text { Erodib. }\end{array}$ \\
\hline$"$ & Ilha Grande & 16,7 & 68,1 & 4,3 & $" \quad "$ & 0,10 \\
\hline$"$ & Santo Antonio do Içá & 60,4 & 18,0 & 9,8 & $" \quad "$ & 0,23 \\
\hline$"$ & Fonte Boa & 33,6 & 44,9 & 2,4 & $"$ & 0,12 \\
\hline$"$ & Santo Antonio do Içá & 9,8 & 84,4 & 3,6 & $" \quad "$ & 0,08 \\
\hline$"$ & Atalaia do Norte & 63,9 & 20,4 & 2,7 & $" \quad "$ & 0,30 \\
\hline$"$ & Humaitá & 45,5 & 37,5 & 5,7 & " " " & 0,21 \\
\hline$"$ & Tapauá & 52,7 & 26,1 & 4,3 & " " " & 0,07 \\
\hline$"$ & Tapauá & 30,3 & 44,6 & 2,8 & $" \quad "$ & 0,13 \\
\hline$"$ & Tapauá & 53,2 & 35,6 & 3,1 & mac. & 0,34 \\
\hline$"$ & Tapauá & 75,6 & 2,5 & 4,1 & Gr. peq. & 0,24 \\
\hline$"$ & Manicoré & 27,1 & 61,3 & 2,6 & " " " & 0,13 \\
\hline$"$ & Novo Aripuanã & 30,3 & 10,8 & 4,1 & & 0,06 \\
\hline$"$ & Lábrea & 68,7 & 9,4 & 5,0 & $"$ & 0,25 \\
\hline$"$ & Manicoré & 67,6 & 16,8 & 4,1 & " " " & 0,26 \\
\hline$"$ & Manicoré & 14,2 & 49,0 & 3,6 & $"$ & 0,07 \\
\hline$"$ & Manicoré & 35,0 & 11,2 & 3,4 & ", & 0,10 \\
\hline$"$ & Manicoré & 34,3 & 50,2 & 4,5 & $" \quad "$ & 0,14 \\
\hline$"$ & Novo Aripuanã & 55,9 & 3,2 & 3,4 & ", & 0,18 \\
\hline$"$ & Novo Aripuanã & 47,5 & 28,2 & 4,9 & $"$ & 0,08 \\
\hline $\mathrm{Pl}$ & Coarr & 51,2 & 39,1 & 0,8 & $" \quad$, & 0,39 \\
\hline$"$ & Manicoré & 75,2 & 1,7 & 4,1 & mac. & 0,30 \\
\hline$"$ & Lábrea & 53,4 & 39,4 & 4,6 & $"$ & 0,25 \\
\hline $\mathrm{Ca}$ & Boca do Acre & 54,0 & 11,0 & 0,8 & bsa & 0,30 \\
\hline$"$ & Labrea & 52,5 & 2.10 & 3,6 & Gr. peq. & 0,23 \\
\hline$"$ & Atalaia do Norte & 68,7 & 19,4 & 7,2 & $" \quad "$ & 0,30 \\
\hline$"$ & São Paulo de Olivença & 4,10 & 1,0 & 3,1 & mac. & 0,11 \\
\hline$A Q$ & Humaitá & 43,0 & 44,0 & 4,6 & Gr. peq. & 0,24 \\
\hline$"$ & Humaitá & 21,5 & 75,5 & 0,5 & $" \quad "$ & 0,23 \\
\hline$"$ & Novo Aripuanã & 8,8 & 91,1 & 1,7 & $" \quad "$ & 0,55 \\
\hline$"$ & Ilha Granae & 75,5 & 22,9 & 3,6 & Gr. peq. & 0,50 \\
\hline $\mathrm{Al}$ & Benjamin Constant & 84,8 & 1,6 & 1,0 & Iam & 0,60 \\
\hline$"$ & IIha Cleto & 53,2 & 3,9 & 1,9 & mac. & 0,20 \\
\hline$"$ & São Pauío ae Olivençá & 25,5 & $66, \bar{z}$ & 3,3 & Gr. peq. & 0,11 \\
\hline GPH & Nova Olincia do Norte & 77,9 & 2,4 & 1,5 & bsa & 0,46 \\
\hline$"$ & Eoca do Acre & 75,0 & 21,0 & 2,6 & mac. & 0,48 \\
\hline$"$ & Atalaia do Norte & 78,6 & 0,4 & $3,3(05)$ & $"$ & 0,33 \\
\hline$"$ & São Paulo de Olivença & 65,3 & 3,8 & $3,3(20)$ & bsa & 0,31 \\
\hline$"$ & Coari & 41,8 & 13,3 & 1,5 & mac. & 0,23 \\
\hline$"$ & Canutama & 83,2 & 1,1 & 3.4 & $"$ & 0,42 \\
\hline$"$ & Atalaia do Norte & 43.3 & 0,8 & $2,1(11)$ & $"$ & 0,15 \\
\hline ? & Atalaia do Norte & 61,5 & 1,1 & $4,4(13)$ & Gr. peq. & 0,33 \\
\hline$"$ & Atalaia do Norte & 58,1 & 1,1 & $5,3(45)$ & $" \quad "$ & 0,33 \\
\hline$"$ & Atalaia do Norte & 64,5 & 4,2 & $3,0(04)$ & bsa & 0,35 \\
\hline $\mathrm{CL}$ & Lábrea & $i 4,0$ & 50,0 & 3,4 & Gr. peq. & 0,08 \\
\hline$"$ & Manicoré & 7,9 & 86,3 & 2,2 & Gr. sples & 0,08 \\
\hline LH & Careiro & 68,6 & 13,6 & 4,0 & bsa & 0,31 \\
\hline$"$ & Lábrea & 55,5 & 22,5 & 2,3 & Gr. peq. & 0,31 \\
\hline$\cdots$ & Lábrea & 61,0 & 25,0 & 4,7 & $" \quad "$ & 0,30 \\
\hline$"$ & Benjamin Constant & 76,6 & 1,2 & 3,7 & bsa & 0,34 \\
\hline$"$ & São Paulo de Olivença & 75,9 & 8,5 & 4,4 & $"$ & 0,36 \\
\hline$"$ & Canutama & 79,8 & 5,1 & 0,9 & mac. & 0,55 \\
\hline $\mathrm{PH}$ & São Gabriel da Cachoeira & 8,4 & 90,2 & 2,7 & Gr. sples & 0,08 \\
\hline$"$ & Humaitá & 13,0 & 86,0 & 0,3 & $" \quad$, & 0,08 \\
\hline
\end{tabular}

(") bsa $=$ blocos subangulares; $\operatorname{mac}=$ maciça; $\mathrm{gr}=$ gra nular $/$ grãos; peq $=$ pequena; $\mathrm{sples}=$ simples. 
Os resultados obtidos com as amostras superficiais dos solos são sugestivos de presença de uma erodibilidade muito baixa no Latossolo Amarelo, Latossolo Vermelho Amarelo, Concrecionário Laterítico e Podzol Hidromórfico; erodibilidade baixa no Podzólico Vermelho Amarelo, e nos Alúvios, e erodibilidade média no Planossolo, Laterita Hidromórfica $\theta$ Glei Pouco Húmico.

No Quadro 4, são apresentados os resultados obtidos com as amostras dos horizontes de perfis de solos Latossólicos e Podzólicos. Na Figura 1, podem ser observadas as seguintes tendências :

QUADRO 3 - Freqüência das classes de erodibilidade revelada pelo porcentual de ocorrência dos valores K.

\begin{tabular}{|c|c|c|c|c|c|c|}
\hline \multirow{2}{*}{\multicolumn{2}{|c|}{ Solos }} & \multicolumn{5}{|c|}{ Classe de erodibilidade } \\
\hline & & Muito Alta & Alta & Média & Baixa & Muito Baixa \\
\hline Latossolo Amarelo & & - & - & - & 12 & 88 \\
\hline Latossolo Vermelho Ame & elo & - & - & - & 34 & 66 \\
\hline Podzólico Vermelho Ame & elo & - & 7 & 17 & 48 & 28 \\
\hline Planossolo & & - & 30 & 70 & - & - \\
\hline Cambissolo & & - & - & 50 & 50 & - \\
\hline Areias Quartzozas & & 50 & - & - & 50 & - \\
\hline Aluvios & & 30 & - & - & 70 & - \\
\hline Glei Pouco Húmico & & - & 30 & 50 & 20 & - \\
\hline Concrecionário Laterítico & & - & - & - & - & 100 \\
\hline Laterita Hidromórfica & 8,0 & - & 20 & 80 & - & - \\
\hline Podzol Hidromórfico & 8,5 & - & - & - & - & 100 \\
\hline
\end{tabular}
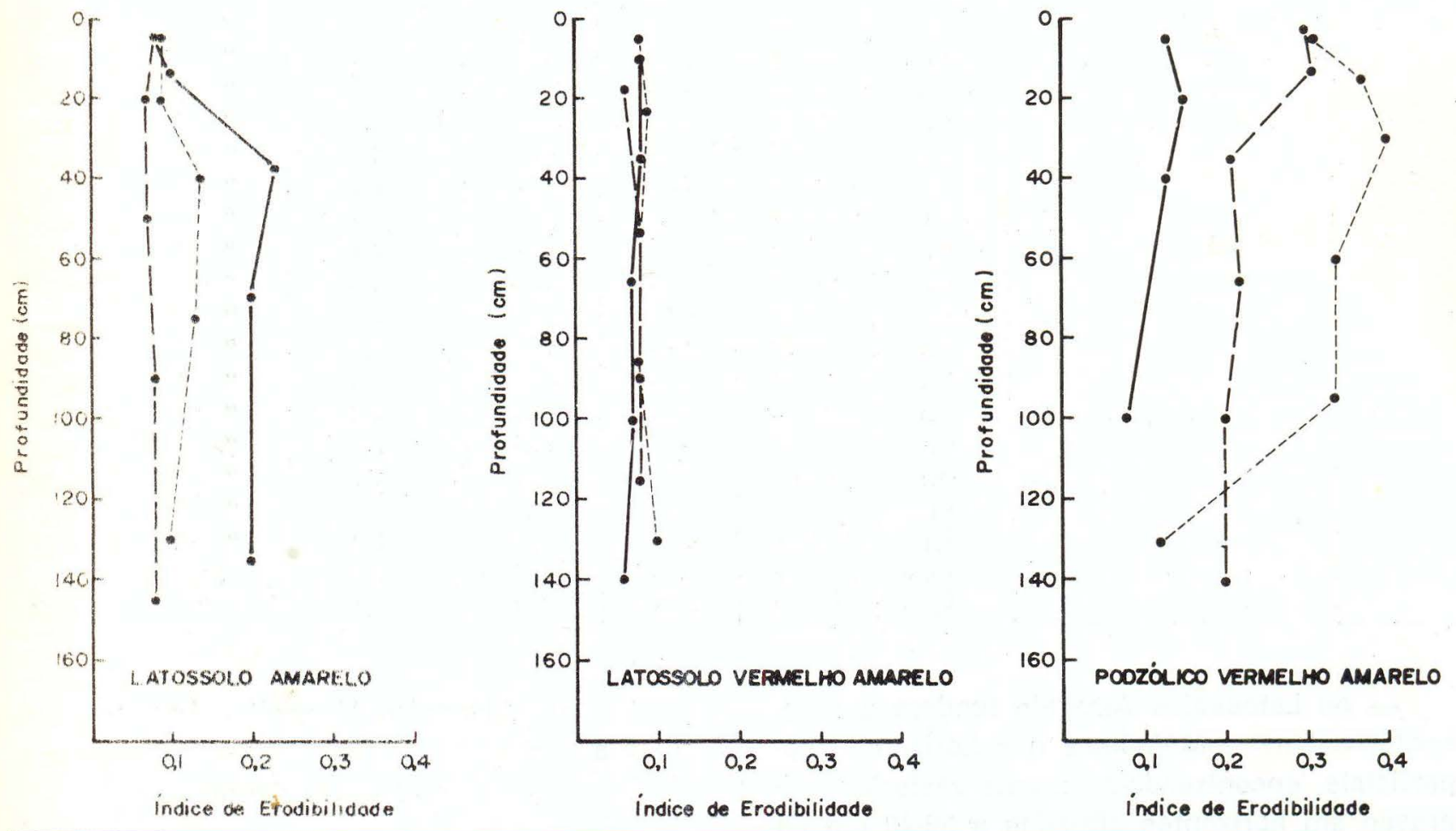

Fig. 1 - Distribuição do índice de Erodibilidade em profundidade nos perfis de solo. 
QUADRO 4 - Resultados obtidos sobre amostras de horizontes de perfis de solo do Estado do Amazonas.

\begin{tabular}{|c|c|c|c|c|c|c|c|c|}
\hline Solo & Município & $\begin{array}{l}\text { Hori- } \\
\text { zonte }\end{array}$ & $\begin{array}{l}\text { Profun- } \\
\text { didade } \\
(\mathrm{cm})\end{array}$ & $\begin{array}{c}\text { Silte } \\
+ \\
\text { s.m.f. }\end{array}$ & $\begin{array}{c}\text { Areia } \\
> \\
0,10 \mathrm{~mm}\end{array}$ & $\begin{array}{c}\text { Matéria } \\
\text { Orgânica }\end{array}$ & Estrutura & $\begin{array}{l}\text { Indice } \\
\text { de } \\
\text { Erodib. }\end{array}$ \\
\hline \multirow[t]{5}{*}{ LA } & \multirow[t]{5}{*}{ Manicoré } & $A_{1}$ & $0-10$ & 18,1 & 62,2 & 5,5 & \multirow{2}{*}{ Gr. peq. } & 0,08 \\
\hline & & $\mathrm{A}_{3}$ & -25 & 15,5 & 56,1 & 1,7 & & 0,10 \\
\hline & & $\mathrm{B}_{1}$ & -50 & 17,7 & 45,1 & 0,5 & \multirow{2}{*}{ mac. } & 0,23 \\
\hline & & $\mathrm{B}_{21}$ & -90 & 13,3 & 48,9 & 0,3 & & 0,20 \\
\hline & & $\mathrm{B}_{22}$ & -180 & 15,4 & 48,5 & 0,2 & $"$ & 0,20 \\
\hline \multirow[t]{5}{*}{ LA } & \multirow[t]{5}{*}{ Novo Aripuanã } & $A_{1}$ & $0-10$ & 31,6 & 31,4 & $-4,6$ & \multirow{2}{*}{$\begin{array}{c}\text { Gr. peq. } \\
"\end{array}$} & 0,09 \\
\hline & & $A_{3}$ & -30 & 11,3 & 31,1 & 1,7 & & 0,09 \\
\hline & & $\mathrm{B}_{1}$ & -50 & 15,8 & 20,8 & 0,9 & \multirow{2}{*}{ mac. } & 0,14 \\
\hline & & $\mathrm{B}_{21}$ & -100 & 17,5 & 15,2 & 0,7 & & 0,13 \\
\hline & & $\mathrm{B}_{22}$ & -160 & 12,8 & 16,1 & 0,3 & " & 0,10 \\
\hline \multirow[t]{5}{*}{ LA } & \multirow[t]{5}{*}{ Novo Aripuanã } & $A_{1}$ & $0-10$ & 34,7 & 6,8 & 5,7 & \multirow{2}{*}{ Gr. peq. } & 0,08 \\
\hline & & $\mathrm{A}_{3}$ & -30 & 8,3 & 20,1 & 2,6 & & 0,07 \\
\hline & & $\mathrm{B}_{1}$ & -70 & 17,9 & 3,7 & 1,4 & & 0,07 \\
\hline & & $\mathrm{B}_{21}$ & -110 & 14,1 & 4,4 & 0,7 & $"$ & 0,08 \\
\hline & & $\mathrm{B}_{22}$ & -180 & 13,0 & 4,2 & 0,5 & $"$ & 0,08 \\
\hline \multirow[t]{5}{*}{ LVA } & \multirow[t]{5}{*}{ Manicoré } & $A_{1}^{2}$ & $0-20$ & 16,1 & 44,0 & 2,7 & & 0,08 \\
\hline & & $A_{3} / B_{1}$ & -50 & 13,7 & 26,8 & 1,2 & & 0,08 \\
\hline & & $\mathrm{B}_{21}$ & -80 & 11,0 & 28,0 & 0,7 & $"$ & 0,07 \\
\hline & & $\mathrm{B}_{22}$ & -120 & 11,5 & 22,7 & 0,5 & & 0,07 \\
\hline & & $\mathrm{B}_{23}$ & -160 & 9,2 & 26,4 & 0,3 & & 0,06 \\
\hline \multirow[t]{5}{*}{ LVA } & \multirow[t]{5}{*}{ Novo Aripuanã } & $A_{1}$ & $0-10$ & 12,3 & 78,6 & 2,8 & $"$ & 0,08 \\
\hline & & $\mathrm{A}_{3}$ & -35 & 13,7 & 72,6 & 1,7 & & 0,09 \\
\hline & & $\mathrm{B}_{1}$ & -70 & 11,3 & 72,9 & 0,9 & & 0,08 \\
\hline & & $\mathrm{B}_{21}$ & -110 & 8,2 & 72,5 & 0,5 & $"$ & 0,08 \\
\hline & & $\mathrm{B}_{22}$ & -150 & 14,8 & 66,4 & 0,3 & & 0,10 \\
\hline \multirow[t]{4}{*}{ LVA } & \multirow[t]{4}{*}{ Novo Aripuanã } & $A_{1}$ & $0-35$ & 22,3 & 14,8 & 4,6 & & 0,06 \\
\hline & & $B_{1}$ & -70 & 19,9 & 10,0 & 1,4 & $"$ & 0,08 \\
\hline & & $\mathrm{B}_{21}$ & -100 & 18,4 & 10,0 & 0,7 & & 0,08 \\
\hline & & $\mathrm{B}_{22}$ & -130 & 23,4 & 8,2 & 0,5 & & 0,08 \\
\hline \multirow[t]{4}{*}{ PVA } & \multirow[t]{4}{*}{ Tapauá } & $A_{1}$ & $0-10$ & 30,3 & 44,6 & 2,8 & & 0,13 \\
\hline & & $\mathrm{A}_{3}$ & -30 & 27,3 & 41,4 & 1,5 & & 0,15 \\
\hline & & $\mathrm{B}_{1}$ & -50 & 23,7 & 48,7 & 0,7 & & 0,13 \\
\hline & & $\mathrm{B}_{2}$ & -150 & 22,9 & 34,9 & 0,5 & & 0,08 \\
\hline \multirow[t]{6}{*}{ PVA } & \multirow[t]{6}{*}{ Manicoré } & $A_{1}$ & $0-5$ & 67,6 & 16,8 & 4,1 & & 0,30 \\
\hline & & $A_{3}$ & -20 & 60,0 & 13,6 & 1,9 & & 0,31 \\
\hline & & $\mathrm{B}_{1}$ & -50 & 50,9 & 12,7 & 0,9 & & 0,21 \\
\hline & & $\mathrm{B}_{21}$ & -80 & 44,5 & 19,2 & 0,5 & & 0,22 \\
\hline & & $\mathrm{B}_{22}$ & -120 & 43,6 & 9,7 & 0,2 & & 0,20 \\
\hline & & $\mathrm{B}_{23}$ & -160 & 42,0 & 13,8 & 0,2 & $"$ & 0,20 \\
\hline \multirow[t]{6}{*}{ PVA } & Lábrea & $A_{11}^{2}$ & -20 & 68,7 & 11,4 & 5,0 & & 0,31 \\
\hline & & $A_{12}$ & $0-10$ & 68,0 & 8,2 & 1,7 & & 0,37 \\
\hline & & $\mathrm{B}_{1}$ & -40 & 73,5 & 4,1 & 0,9 & & 0,40 \\
\hline & & $\mathrm{B}_{21}$ & -80 & 65,4 & 4,7 & 0,9 & $" \quad "$ & 0,34 \\
\hline & & $\mathrm{B}_{22} \mathrm{pl}$ & -110 & 64,0 & 5,7 & 0,7 & bsa & 0,34 \\
\hline & & $\mathrm{B}_{23} \mathrm{pl}$ & -150 & 37,2 & 8,8 & 0,7 & " & 0,12 \\
\hline
\end{tabular}

- os Latossolos Amarelo tendem a apresentar os menores índices nos horizontes superficiais, encontrando-se menor resistência à erosão em horizontes situados a $30-40 \mathrm{~cm}$ de profundidade. A partir daí a erodibilidade tende a diminuir com a profundidade;
- os Latossolos Vermelho Amarelos tendem a apresentar índices de erodibilidade muito baixos em toda a extensão do perfil;

- os solos Podzólicos tendem a apresentar índices de erodibilidade mais elevados do 
que os solos Latossólicos, havendo ainda a tendência de um ligeiro aumento da resistência à erosão, com a profundidade.

\section{SUMMARY}

A nomographic method was applied to existing ata on soil great groups of the state of Amazonas, in order to obtain erodibility values. The results show that Red Yellow Podzolic Soils are more susceptible to erosion than Latosolic soils. Red Yellow Latosol seems to be more resistant to erosion than Yellow Latosol, as revealed by lower erodibility values of profile horizons.

Freire, O. \& Pessotti, J.E.

1974 - Erodibilidade dos solos do Estado de São Paulo. Anais da E.S.A. Luiz de Queiróz, 31: 333-350.

Frojeto RADAM BRASIL

1975/78 - Levantamento de Recursos Naturais. Vols. 8, 9, 10, 11, 12, 13, 14, 15, 16 e 17. Rio de Janeiro, Min. Minas e Energia.
RANZANI, G.

1980 - Identificação e Caracterização de Alguns Solos da Estação Experimental de Silvicultura Tropical do INPA. Acta Amazonica, 10 (1).

SMITH, R.M. \& ABRUNA, F.

1955 - Soil and Water Conservation Research in Puerto Rico 1938 to 1947. Univ of Puerto Ríco Agr. Exp. 5 ta. Bul., (125).

Wischmeier, W.H.; Johnson, C.B. \& Cross, B.V.

1971 - A Soil Erodibility Nomograph for Farmland and Construction Sites. Journ. of Soil and Water Conserv., 26 (5): 189-193.

WISCHMEIER, W.H. \& SMITH, D.D.

1965 - Predicting Rainfall - Erosion Losses from Cropland East of the Rocky Mountains. Agric. Handbook, USDA (282).

(Aceito para publicação em 29/01/80) 